ISSN 1997-342X (Online), ISSN 1991-8631 (Print)

Original Paper http://ajol.info/index.php/ijbcs http://indexmedicus.afro.who.int

\title{
Caractérisation hydrochimique de la nappe de Yao et ses environs : relations entre eaux de surface (lac-Fitri) et eaux souterraines
}

\author{
Bichara LOUKMAN ${ }^{1 *}$, Samuel NAKOLENDOUSSE ${ }^{1}$, Abdallah MAHAMAT NOUR ${ }^{2}$ \\ and Mberdoum NGUINAMBAYE MEMTI ${ }^{2}$ \\ ${ }^{1}$ Laboratoire GEORESSOURCES, Unité de Formation et de Recherche/Sciences de la Vie et de la Terre. \\ Université Ouagal Pr. Joseph KI-ZERBO : 03 BP 7023 Ouagadougou 03, Burkina Fasso. \\ ${ }^{2}$ Laboratoire de Géologie, Faculté des Sciences Exactes et Appliquées, Université de N'Djamena, BP 1027, \\ N'Djamena, Tchad. \\ *Auteur correspondant ; E-mail : louknan.bichara@gmail.com
}

\section{REMERCIEMENTS}

Nous remercions l'Etat Tchadien à travers l'Université de N'Djamena pour le financement de ce travail.

\section{RESUME}

Les eaux souterraines constituent l'une des principales sources d'approvisionnement en eau potable de la population du département de Fitri. Dans cette zone, aucune étude hydrochimique significative des eaux souterraines n'a été entreprise. C'est pourquoi, cette étude s'est fixée pour objectif principal de faire l'état de la qualité chimique des eaux de l'aquifère. Elle précise l'origine de la minéralisation dans les eaux souterraines à partir d'une combinaison des méthodes hydrochimiques et de l'analyse statistique multivariée. Les analyses physicochimiques ont été effectuées sur 21échantillons d'eau de pompes de villages autours de Yao et l'eau du lac Fitri. Cette étude montre que les eaux souterraines exploitées dans cette zone ont une température moyenne de $27.90{ }^{\circ} \mathrm{C}$. Elles varient de $24,87{ }^{\circ} \mathrm{C}$ à Maskao et de $32,17^{\circ} \mathrm{C}$ à Amdjoudoula, Syala, Mafé, N'DjamenaBilala. Ces valeurs semblent être régies par les réactions physico-chimiques exothermiques telles que l'oxydation et la réduction. Dans l'ensemble, des eaux échantillonnées, les $\mathrm{pH}$ mesurés sont peu hétérogènes et se situent autour de la neutralité. Il varie de 6,34 à 8,13 avec une moyenne de 7,02. Les valeurs les plus faibles sont observées à Beptongo, Goubichou et Kilguim et les plus élevées à Abrania. Les valeurs de la conductivité électrique sont très hétérogènes et sont comprises entre $122 \mu \mathrm{S} / \mathrm{cm}$ et $18740 \mu \mathrm{S} / \mathrm{cm}$. Cette conductivité qui augmente dans le sens de l'écoulement permet de conclure qu'il s'agit de l'influence de la nature lithologique plus ou moins évaporitiques. L'analyse en Classification Hiérarchique Ascendante (CHA) a permis d'identifier quatre groupes d'eau de compositions chimiques différentes.

(C) 2017 International Formulae Group. All rights reserved.

Mots clés : Eaux souterraines, minéralisation, Hydrochimie, recharge, Batha, Yao Fitri. 


\title{
Hydrochimical characterization of the tablecloth of Yao and its surroundings: relation between surface water and subsoil waters
}

\begin{abstract}
The subsoil waters constitute one of the principal sources of supply drinking water of the population of the department of Fitri. In this zone, no significant hydrochimical study of subsoil waters was undertaken. That is why, this study aimed at giving the report on the chemical quality of water of the aquifer. It specifies the origin of mineralization in subsoil waters starting from a combination of the hydrochimic methods and multivariate statistical analysis. The physic-chemical analyses were carried out on 21 samples water of pumps of village's goshawks of Yao and the water of the lake Fitri. This study shows that the subsoil waters exploited in this zone have an average temperature of $27.90{ }^{\circ} \mathrm{C}$. They range between $24.87 \mathrm{C}$ in Maskao and and 32.17 ${ }^{\circ} \mathrm{C}$ in Amdjoudoula, Syala, Mafé, and Djamena-.Bilala. These values seem to be controlled by the exothermic physicochemical reactions such as oxidation and the reduction. In the whole of sampled water, the $\mathrm{pH}$ measured is not very heterogeneous and are around neutrality. It varies from 6.34 to 8.13 with an average of 7.02. The lowest values are observed in Beptongo, Goubichou and Kilguim and highest with Abrania. The values of electric conductivity are very heterogeneous and lie between $122 \mu \mathrm{S} / \mathrm{cm}$ and $18740 \mu \mathrm{S} / \mathrm{cm}$. This conductivity which increases in the direction of the flow makes it possible to conclude that it is about the influence of lithological nature more or less evaporitic. The analysis in Ascending Hierarchical Classification (CHA) made it possible to identify four groups of water of different chemical compositions.

(C) 2017 International Formulae Group. All rights reserved.
\end{abstract}

Keywords: Subsoil waters, mineralization, Hydrochimic, refill, Batha, Yao Fitri.

\section{INTRODUCTION}

La Région de Batha, située au centre du Tchad, d'une superficie de $84000 \mathrm{~km}^{2}$, est composée de trois départements : le Batha Ouest (Ati), le Batha Est (Oum Hadjer) et le Fitri (Yao) où se trouve la zone d'étude (Figure 1).

Le département du Fitri s'étend entre $12^{\circ} 42^{\prime} 30$ "et $13^{\circ} 2^{\prime} 0^{\prime \prime}$ de latitude Nord et entre $17^{\circ} 26^{\prime} 0^{\prime \prime}$ et $17^{\circ} 57^{\prime} 30$ de longitude Est et couvre une superficie $2088 \mathrm{~km}^{2}$ avec une densité de 13,5 hbts $/ \mathrm{km}^{2}$ (RGPH, 2009). Dans ce département, les conditions climatiques ont des conséquences néfastes sur le milieu naturel où la baisse pluviométrique pèse sur la recharge de la nappe. Le climat est de type intertropical, avec une saison des pluies située entre juin et octobre. La pluviométrie oscille entre 200 à $800 \mathrm{~mm}$ (Baohoutou et al., 2014). Les formations sédimentaires constituent le remplissage de toute la région. $\mathrm{La}$ zone d'étude est caractérisée par une prédominance des dépôts quaternaires notamment une succession de sédiments détritiques sabloargileux du Continental Terminal (CT), du Pliocène et du Quaternaire (Karaki et al., 2006).

Par ailleurs, ce département est confronté à d'énormes difficultés allant de la faible pluviosité et une répartition inégale du Sud au Nord des précipitations. Les longues périodes de sécheresse l'ont affectée durant ces dernières décennies avec comme corollaire l'assèchement du lac Fitri (Djoret, 2000).

Des travaux antérieurs ont été réalisés dans la dépression piézométrique (Massuel, 2001 ; Leblanc, 2002), mais ne permettent pas de comprendre le processus de minéralisation des eaux sur l'ensemble de la zone; ni d'indiquer les origines de la salinité des eaux. Ils ne donnent pas une idée sur les zones de recharge ni sur la relation entre la nappe et les eaux de surface.

En effet, la consommation d'eau à faible teneur en minéraux a un effet négatif 
sur les mécanismes humains. Une étude épidémiologique effectuée dans des secteurs alimentés par de l'eau dure (plus de 400-500 $\mathrm{mg} / \mathrm{l}$ de $\mathrm{CaCO}_{3}$ ) pouvait être la cause de taux plus élevés de certaines maladies incluant le cancer (Lara Fabriz et al., 2004). On constate que la population de cette zone ne cesse de se plaindre des maladies urinaires ou autres, suite à la consommation des eaux de pompes. Mais, pas de preuve à cet instant disponible pour documenter l'aspect nocif pour la santé de l'eau de pompe dans notre zone d'étude. Nous poursuivrons nos recherches en profondeur ultérieurement.

Peut-être seulement, une forte teneur en magnésium (des centaines de mg/l) couplée avec une forte teneur en sulfate peut entraîner des diarrhées. Par conséquent, les questions d'alimentation en eau potable restent des problèmes de santé publique.

$\mathrm{Au}$ regard de tous ces problèmes, il sera judicieux de mener des études approfondies pour mieux connaître les charges en ions des eaux de forages ou des puits. Ceci permettra de connaître l'origine de la minéralisation.

\section{MATERIEL ET METHODES}

\section{Localisation de la zone d'étude}

La Région de Batha, située au centre du Tchad, d'une superficie de $84000 \mathrm{~km}^{2}$, est composée de trois départements : le Batha Ouest (Ati), le Batha Est (Oum Hadjer) et le Fitri (Yao) où se trouve la zone d'étude. Ce département de Fitri se trouve dans le bassin hydrologique du lac Tchad et s'étend entre $12^{\circ} 23^{\prime}$ et $15^{\circ} 35^{\prime}$ de latitude Nord et entre $17^{\circ} 03^{\prime}$ et $20^{\circ} 28^{\prime}$ de longitude Est. Il couvre une superficie $2088 \mathrm{~km}^{2}$ avec une densité de $13,5 \mathrm{hbts} / \mathrm{km}^{2}$ sur laquelle vivent 116.157 habitants (RGPH, 2009).

\section{Matériel}

Au cours de ce travail de recherche, on a utilisé sur le terrain:

Le GPS GARMIN 72 pour la prise des coordonnées géographiques des points d'eau mesurés et échantillonnés; la Sonde électrique pour faire les relevés piézométriques; le décamètre pour effectuer des mesures sur les margelles des puits; une carte topographique 1/200 000 permet de positionner et d'orienter vers un point ciblé ; des fiches techniques des points d'eau; sur lesquelles les informations sont consignées; des bouteilles d'échantillonnages, le Kit multi-paramètre WTW pour les mesures des paramètres physico-chimiques in situ et un Appareil photo numérique a servi de faire des photos à la zone d'étude pour illustrer le mémoire.

\section{Mesures sur le terrain et échantillonnage}

Les données qui constituent l'objet de ce travail proviennent des puits et forages et du lac Fitri dont on dispose les paramètres hydrodynamiques.

\section{Niveau statique}

Le niveau statique désigne le plan d'eau relevé, stabilisé ou non, dans un puits de pompage ou un ouvrage d'injection (Scuster, 2004).

\section{Conductivité électrique}

La conductivité électrique traduit la capacité d'une eau (solution aqueuse) à conduire le courant électrique, elle est directement liée à la force ionique qui découle des formations traversées par cette eau dans le bassin hydrogéologique ainsi qu'à son temps de séjour dans le réservoir. Ce paramètre a été mesuré à l'aide d'un conductivimètre de marque WTW en plongeant les deux électrodes dans un récipient contenant de l'eau à examiner.

\section{Potentiel d'Hydrogène (pH)}

Le $\mathrm{pH}$ est la mesure de l'acidité de l'eau. Il exprime la concentration en ions hydrogène $\mathrm{H}^{+}$d'une eau. Les caractères plus acide (inférieur à 5) ou plus basique (supérieur à 8,5) altèrent les micro-organismes vivant dans une eau. Dans nos travaux, le $\mathrm{pH}$ des 
échantillons a été mesuré à l'aide d'un $\mathrm{pH}$ mètre de marque WTW en plongeant l'électrode dans un bécher contenant $100 \mathrm{ml}$ environ d'échantillon.

\section{Campagne d'échantillonnage}

L'ensemble de la région possède une même nappe phréatique générale, mais les terrains qui la recèlent présentent des faciès très variés du sable dénué de fines à l'argile imperméable, ce qui entraine une grande hétérogénéité dans les caractéristiques hydrauliques et chimiques de la nappe (Schneider, 2001).

La première campagne s'est déroulée pendant les mois d'août-septembre 2016. Lors de cette campagne, des échantillons de lac et d'eaux souterraines ont été récoltés. A chaque fois, les paramètres physico-chimiques ont été mesurés in-situ et des prélèvements pour les éléments majeurs ont été réalisés. Le suivi du fleuve Batha et ses affluents a été échantillonné. Deux (2) points du lac et 21 points d'aquifères ( 9 puits et 11 forages) ont été récoltés autours du lac dont ils font l'objet des analyses physico-chimique et chimique.

Les échantillons ont été prélevés dans des bouteilles en plastique (1,5 litres) extrêmement propres et rincées 3 fois avec de l'eau à analyser. Pour .le forage à pompe manuelle, un pompage à longue durée a été effectué. Les échantillons ont été remplis à ras de bord et fermés de façon totalement étanche pour éviter les bulles d'air dans le flacon qui pourraient favoriser le dégagement de certains gaz dissous dans l'eau, ce qui modifierait les équilibres chimiques dans la solution (variation du $\mathrm{pH}$ oxydation avec l'oxygène de l'air, etc.). Tous ces échantillons ont été conservés à froid puis acheminés au laboratoire national des Eaux (LNE) du ministère de l'élevage et de l'hydraulique pour l'analyse chimiques.

Les analyses de sodium et potassium par le Photomètre à flamme. Celles de sulfates et de nitrate ont été faites par le Spectrophotomètre à flamme (DR2800). Le Système de titration Hach pour la mesure de l'alcalinité (ion bicarbonate). La mesure du paramètre magnésium a été faite par la soustraction entre la valeur du calcium avec celle de la dureté totale $(\mathrm{TH})$.

Au cours de ces analyses, nous avons utilisé des réactifs spécifiques pour les analyses de différents paramètres.

Pour la mesure du Nitrate, le tube de l'échantillon contient le réactif Nitraver a été placé dans le spectrophotomètre (DR2800) calibré à priori en introduisant un tube contenant $25 \mathrm{ml}$ de l'échantillon à blanc. Pour ce qui est de l'ion bicarbonate, l'indicateur HELIANTHINE a été introduit puis titré avec de l'acide sulfurique de normalité $0.02 \mathrm{~N}$. La mesure du Chlore, l'indicateur CHROMATE de potassium $\left(\mathrm{K}_{2} \mathrm{CrO}_{4}\right)$ est introduit dans 100 $\mathrm{ml}$ de l'échantillon à analyser puis titré avec du nitrate d'argent $\left(\mathrm{AgNO}_{3}\right)$.

\section{Le diagramme de Piper}

Le diagramme de Piper (Figure 2) (Simler, 2013), met en évidence l'incidence de la géologie sur la qualité des eaux. Il permet aussi d'estimer les pourcentages des éléments chimiques et leur classification (Fehdi Chemseddine et al., 2009). Il a révélé que les eaux sont à environ $89 \%$ bicarbonaté calcique dont plus de $80 \%$ chlorurées sodipotassiques. En effet, ce diagramme permet de représenter sur un même graphique des nombreuses analyses autorisant des regroupements des faciès chimiques.

\section{Analyses statistiques \\ Le Logiciel Arc GIS 10 a été utilisé pour l'extraction des côtes du sol (Z) des différents points d'ouvrage et le Logiciel de dessin (Surfer) a permis de réaliser des courbe hydro isohypses et la carte de vue $3 \mathrm{D}$ du bassin hydrogéologique. Le Logiciel Tableur Excel a servi au calcul de la cote piézométrique $(\mathrm{Zp})$ des ouvrages.}




\section{LOCALISATION DU DEPARTEMENT DE FITRI}

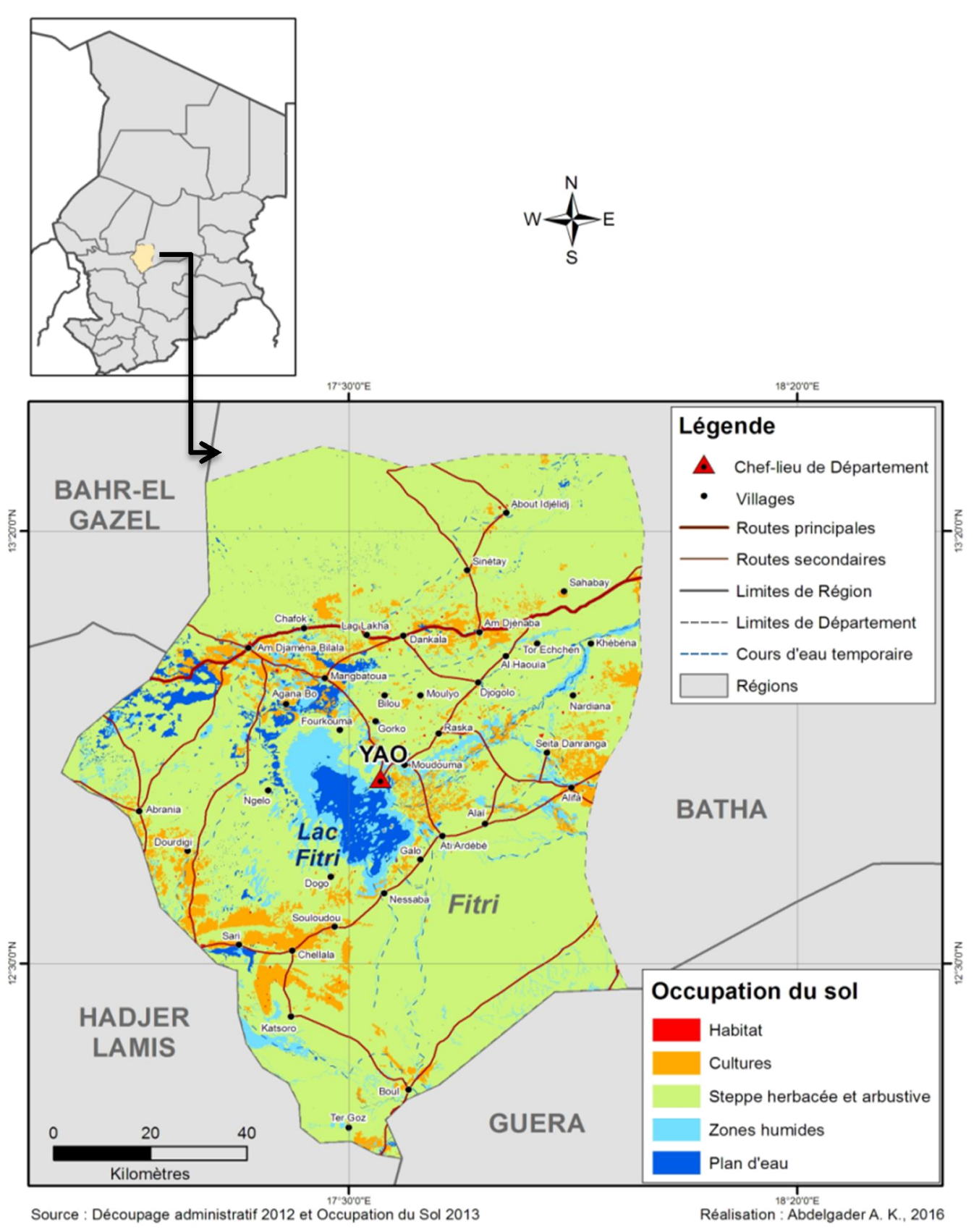

Figure 1: Localisation du site d'étude. 


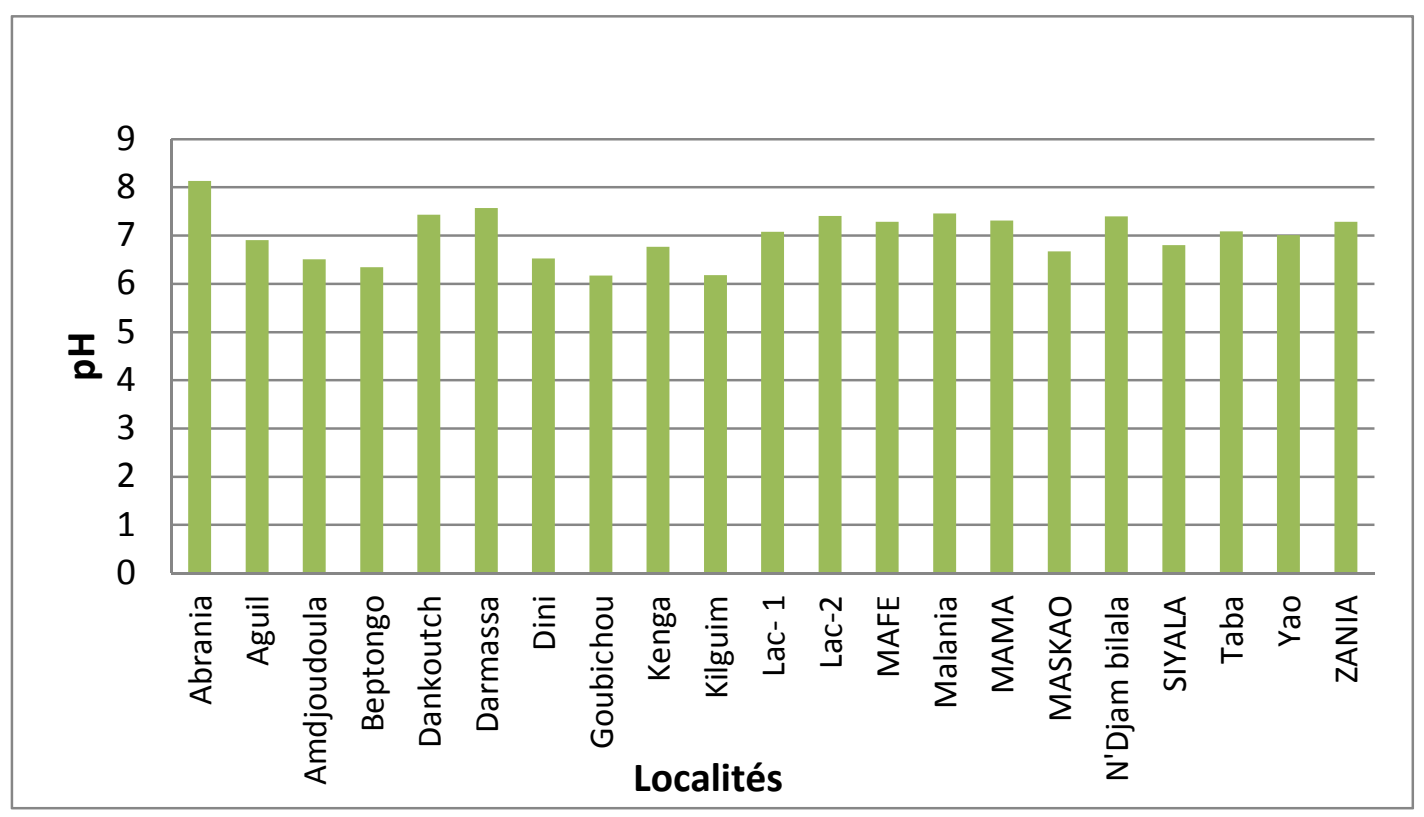

Figure 2 : Histogramme de $\mathrm{pH}$ mesuré.

\section{RESULTATS}

\section{Paramètres hydrodynamiques}

\section{Niveau statique et piézométrique}

Les paramètres indiqués dans le Tableau 1 ont permis d'établir la carte piézométrique de la zone d'étude. Ainsi, le niveau piézométrique le plus élevé se situe à Zania. Par contre, le niveau le plus bas se trouve à Kenga.

La carte piézométrique (Figure 3) met en évidence l'orientation de sens d'écoulement, les zones de recharges et des décharges.

\section{Conductivité}

La conductivité électrique des eaux est le reflet de la minéralisation totale. Les valeurs de la conductivité électrique de la zone d'étude sont très hétérogènes et sont comprises entre $122 \mu \mathrm{S} / \mathrm{cm}$ et $18480 \mu \mathrm{S} / \mathrm{cm}$. Les plus faibles sont mesurées à Aguil, Mafé, Zania et N'Djamena Bilala et les plus élevées se situent à Beptongon, Abrania et Goubichou. La Figure 4 montre des conductivités très élevées dépassant 1000 $\mu \mathrm{S} / \mathrm{cm}$.

\section{Potentiel d'hydrogène (pH)}

Dans l'ensemble des eaux échantillonnées, les $\mathrm{pH}$ mesurés sont peu hétérogènes et se situent autour de la neutralité. Ils varient entre 6,34 à 8,13 avec une moyenne de 7,02. Les valeurs les plus faibles sont observées dans la zone de Beptongo, Goubichou et Kilguim et les plus élevées dans les zones d'Abrania. Cette augmentation serait due à une progression du faciès des eaux qui passe de bicarbonaté calcique au bicarbonaté sodique (Fréderic, 2000, Schuster et al., 2016).

\section{Température}

La température moyenne des eaux souterraines est de $27,90^{\circ} \mathrm{C}$. Elles varient de $24,87^{\circ} \mathrm{C}$ les plus basses à Maskao, à $32,17^{\circ} \mathrm{C}$ les plus élevées à Amdjoudoula.

\section{Eléments chimiques majeurs}

La minéralisation des eaux souterraines est contrôlée par certains ions, notamment les 
cations (le calcium, le magnésium, le potassium et le sodium) et les anions (le bicarbonate, le sulfate, le chlorure, et le nitrate). Ainsi, les concentrations en poids de ces éléments majeurs pour les cations, consignées dans le (Tableau 2), montrent une variation d'un échantillon à l'autre. Les concentrations pondérales en calcium varient de $12 \mathrm{mg} / \mathrm{l}$ (Mafée) à $19485 \mathrm{mg} / \mathrm{l}$ à (Kilguim), le magnésium de 1,2 mg/l lac Fitri 2 à $510,2 \mathrm{mg} / 1$ (Taba), le potassium varie de $1,2 \mathrm{mg} / \mathrm{l}$ (Goubichou) à 57,8 mg/l (Lac-Fitri 1) et le sodium de $12 \mathrm{mg} / \mathrm{l}$ (Mafé) à $3251 \mathrm{mg} / 1$ (Kilguim).

Les concentrations pondérales des échantillons pour les anions montrent une hétérogénéité en bicarbonates qui varient respectivement de 73,2 $\mathrm{mg} / \mathrm{l}$ (Mafée) à 3050 $\mathrm{mg} / \mathrm{l}$ (Taba), Les teneurs en nitrates, varient de $1,3 \mathrm{mg} / \mathrm{l}$ (Kenga) à $12 \mathrm{mg} / \mathrm{l}$ (Darmassa). Par contre, celles de chlorures et en sulfates varient de $11 \mathrm{mg} / \mathrm{l}$ (Mafé) à $1652 \mathrm{mg} / \mathrm{l}$ (Kilguim) et 5 mg/l (Dini) à 3647 mg/l (Taba).

\section{Le diagramme de Piper}

La projection des résultats des analyses chimiques dans ce diagramme fait apparaître clairement une très grande variation de la chimie des eaux souterraines: Faciès bicarbonaté calcique, Faciès bicarbonaté sodique et Faciès sulfaté sodique.

Tableau 1: Niveau statique et Niveau piézométrique.

\begin{tabular}{|c|c|c|c|c|c|c|}
\hline $\mathbf{N}^{\circ}$ & Localités & $\mathbf{X}$ & $\mathbf{Y}$ & $\mathrm{Z}(\mathbf{m})$ & Ns(m) & $\mathbf{N p}(\mathrm{m})$ \\
\hline 1 & Abrania & $17^{\circ} 36^{\prime} 23^{\prime \prime}$ & $12^{\circ} 58^{\prime} 24^{\prime \prime}$ & 360,2 & 13,7 & 346,5 \\
\hline 2 & Aguil & $17^{\circ} 51^{\prime} 14^{\prime \prime}$ & $12^{\circ} 52^{\prime} 45^{\prime \prime}$ & 347,2 & 25,7 & 321,5 \\
\hline 3 & Amdjoudoula & $12^{\circ} 28^{\prime} 45^{\prime \prime}$ & $17^{\circ} 43^{\prime} 62^{\prime \prime}$ & 369,5 & 22,5 & 347 \\
\hline 4 & Beptongo & $17^{\circ} 44^{\prime} 00^{\prime \prime}$ & $12^{\circ} 53^{\prime} 18^{\prime \prime}$ & 354,6 & 11,3 & 343,3 \\
\hline 5 & Dankoutch & $17^{\circ} 53^{\prime} 51^{\prime \prime}$ & $12^{\circ} 59^{\prime} 03^{\prime \prime}$ & 353,4 & 15,5 & 337,9 \\
\hline 6 & Darmassa & $17^{\circ} 37^{\prime} 59^{\prime}$, & $12^{\circ} 58^{\prime} 38^{\prime \prime}$ & 329,2 & 23,8 & 305,4 \\
\hline 7 & Dini & $17^{\circ} 32^{\prime} 21^{\prime \prime}$ & $12^{\circ} 53^{\prime} 18^{\prime \prime}$ & 308,7 & 17,3 & 291,4 \\
\hline 8 & Goubichou & $17^{\circ} 47^{\prime} 29^{\prime \prime}$ & $12^{\circ} 51^{\prime} 45^{\prime \prime}$ & 327,7 & 23,2 & 304,5 \\
\hline 9 & Kenga & $17^{\circ} 32^{\prime} 20^{\prime \prime}$ & $12^{\circ} 57^{\prime} 59^{\prime \prime}$ & 298,7 & 19,2 & 279,5 \\
\hline 10 & Kilguim & $17^{\circ} 36^{\prime} 29^{\prime \prime}$ & $12^{\circ} 56^{\prime} 49^{\prime \prime}$ & 329,6 & 31,3 & 298,3 \\
\hline 11 & Lac 1 & $17^{\circ} 33^{\prime} 42^{\prime \prime}$ & $12^{\circ} 49^{\prime} 08^{\prime \prime}$ & 344 & 0,8 & 343,2 \\
\hline 12 & Lac 2 & $17^{\circ} 32^{\prime} 58^{\prime \prime}$ & $12^{\circ} 49^{\prime} 20^{\prime \prime}$ & 344 & 0,8 & 343,2 \\
\hline 13 & MAFE & $17^{\circ} 40^{\prime} 12^{\prime \prime}$ & $12^{\circ} 51^{\prime} 10^{\prime \prime}$ & 361,7 & 15,2 & 346,5 \\
\hline 14 & Malania & $17^{\circ} 51^{\prime} 38^{\prime \prime}$ & $12^{\circ} 51^{\prime} 02^{\prime \prime}$ & 341,2 & 25,8 & 315,4 \\
\hline 15 & MAMA & $17^{\circ} 36^{\prime} 12^{\prime \prime}$ & $13^{\circ} 00^{\prime} 28^{\prime \prime}$ & 322,5 & 24,01 & 298,49 \\
\hline 16 & MASKAO & 17³9'54', & $12^{\circ} 58^{\prime} 35^{\prime \prime}$ & 354,6 & 22,4 & 332,2 \\
\hline 17 & N'Djamena Bilala & $17^{\circ} 18^{\prime} 92^{\prime \prime}$ & $13^{\circ} 06^{\prime} 19^{\prime}$, & 345 & 13,25 & 331,75 \\
\hline 18 & SIYALA & $17^{\circ} 40^{\prime} 09^{\prime \prime}$ & $12^{\circ} 53^{\prime} 25^{\prime \prime}$ & 308,1 & 9,9 & 298,2 \\
\hline 19 & Taba & $17^{\circ} 39^{\prime} 16^{\prime \prime}$ & $12^{\circ} 55^{\prime} 44^{\prime \prime}$ & 347 & 22,9 & 324,1 \\
\hline 20 & Yao & $17^{\circ} 33^{\prime} 40^{\prime \prime}$ & $12^{\circ} 50^{\prime} 58^{\prime \prime}$ & 337,3 & 7,6 & 337,3 \\
\hline 21 & ZANIA & $17^{\circ} 45^{\prime} 32^{\prime \prime}$ & $12^{\circ} 54^{\prime} 44^{\prime \prime}$ & 357 & 5 & 352 \\
\hline
\end{tabular}


B. LOUKMAN et al. / Int. J. Biol. Chem. Sci. 11(3): 1336-1349, 2017

Tableau 2: Cations, Anions et paramètres physico- chimiques (en mg/l).

\begin{tabular}{|c|c|c|c|c|c|c|c|c|c|c|c|c|c|c|}
\hline \multirow{2}{*}{ Villages } & \multicolumn{5}{|c|}{ Paramètres physicochimiques } & \multicolumn{4}{|c|}{ Cations en mg/L } & \multicolumn{4}{|c|}{ Anions en mg/L } & \multirow[b]{2}{*}{ Balance ionique } \\
\hline & pH & Ecart type & $\mathbf{T}^{\circ} \mathbf{C}$ & Ecart type & $\mathrm{EC}(\boldsymbol{\mu S} / \mathbf{C m})$ & $\mathbf{C a}$ & Mg & $\mathbf{K}$ & $\mathbf{N a}$ & HCO3 & SO4 & NO3 & Cl & \\
\hline Abrania & 8,13 & 0,35 & 27,00 & 0,53 & 12158 & 250 & 135,2 & 18,5 & 1150 & 1221,1 & 2112 & 3,2 & 602 & $-4,38$ \\
\hline Aguil & 6,91 & 0,02 & 28,43 & 0,25 & 122 & 210 & 3,2 & 2,2 & 45 & 452,2 & 25 & 4,1 & 216 & 7,93 \\
\hline Amdjoudoula & 6,51 & 0,06 & 32,17 & 0,76 & 1120 & 259 & 3,01 & 7,1 & 261 & 709 & 623 & 8 & 45 & $-2,49$ \\
\hline Beptongo & 6,34 & 0,01 & 28,57 & 0,38 & 18480 & 480 & 1,4 & 5,3 & 467 & 1327 & 365 & 9 & 398 & 4,5 \\
\hline Dankoutch & 7,43 & 0,32 & 26,77 & 0,25 & 678 & 1100 & 5,2 & 38 & 788 & 2599,1 & 1758 & 7 & 378 & 0,37 \\
\hline Darmassa & 7,57 & 0,21 & 26,37 & 0,15 & 720 & 170 & 70 & 30,5 & 435 & 985 & 314 & 12 & 342 & 2,27 \\
\hline Dini & 6,53 & 0,06 & 26,77 & 0,25 & 810 & 22 & 10,1 & 3,5 & 34 & 146,4 & 5 & 4,1 & 28 & 2,19 \\
\hline Goubichou & 6,17 & 0,03 & 28,30 & 0,17 & 14120 & 783 & 2 & 1,2 & 133 & 1121,2 & 66 & 2,3 & 924 & $-0,68$ \\
\hline Kenga & 6,77 & 0,25 & 26,20 & 0,00 & 710 & 69 & 15,2 & 43 & 68 & 366 & 32 & 1,3 & 60 & 2,32 \\
\hline Kilguim & 6,18 & 0,03 & 28,23 & 0,40 & 3850 & 1985 & 45 & 43 & 3251 & 2100 & 3250 & 3,1 & 1652 & 24,54 \\
\hline Lac- 1 & 7,08 & 0,27 & 27,10 & 0,21 & 179 & 280 & 38,9 & 57,8 & 993 & 1025 & 1380 & 3,6 & 520 & 0,9 \\
\hline Lac-2 & 7,41 & 0,31 & 26,30 & 0,26 & 127 & 42,4 & 1,2 & 4,4 & 6,3 & 97,6 & 6 & 3 & 31 & $-0,76$ \\
\hline MAFE & 7,29 & 0,04 & 30,87 & 0,91 & 320 & 12 & 8,7 & 4,8 & 12 & 73,2 & 12 & 9 & 11 & 1,67 \\
\hline Malania & 7,46 & 0,31 & 26,63 & 0,21 & 1214 & 435 & 36 & 11,5 & 355 & 1036,5 & 789 & 6 & 130 & 4,23 \\
\hline MAMA & 7,31 & 0,26 & 28,30 & 0,10 & 1089 & 263 & 2,5 & 6,9 & 252 & 806 & 81 & 8 & 283 & 3,13 \\
\hline MASKAO & 6,67 & 0,23 & 24,87 & 0,12 & 820 & 226 & 39 & 28 & 127 & 679 & 76 & 2,8 & 256 & 2,01 \\
\hline N'Djam bilala & 7,40 & 0,02 & 30,90 & 0,10 & 240 & 321 & 54 & 4,5 & 287 & 410 & 489 & 3,4 & 478 & 4,27 \\
\hline SIYALA & 6,80 & 0,00 & 32,53 & 0,45 & 1125 & 259 & 2,5 & 6,2 & 248 & 812 & 78,5 & 7,5 & 286 & 2,06 \\
\hline Taba & 7,09 & 0,26 & 28,40 & 0,20 & 820 & 980 & 510,2 & 27 & 1542 & 3050 & 3647 & 7 & 1098 & 0,7 \\
\hline Yao & 7,01 & 0,21 & 27,2 & 0,54 & 928 & 250 & 5,1 & 9,5 & 245 & 886,2 & 53 & 6 & 214 & 4,59 \\
\hline ZANIA & 7,29 & 0,10 & 26,57 & 0,38 & 195 & 589 & 58 & 4,9 & 78 & 1123 & 623 & 1,8 & 182 & 1,68 \\
\hline
\end{tabular}




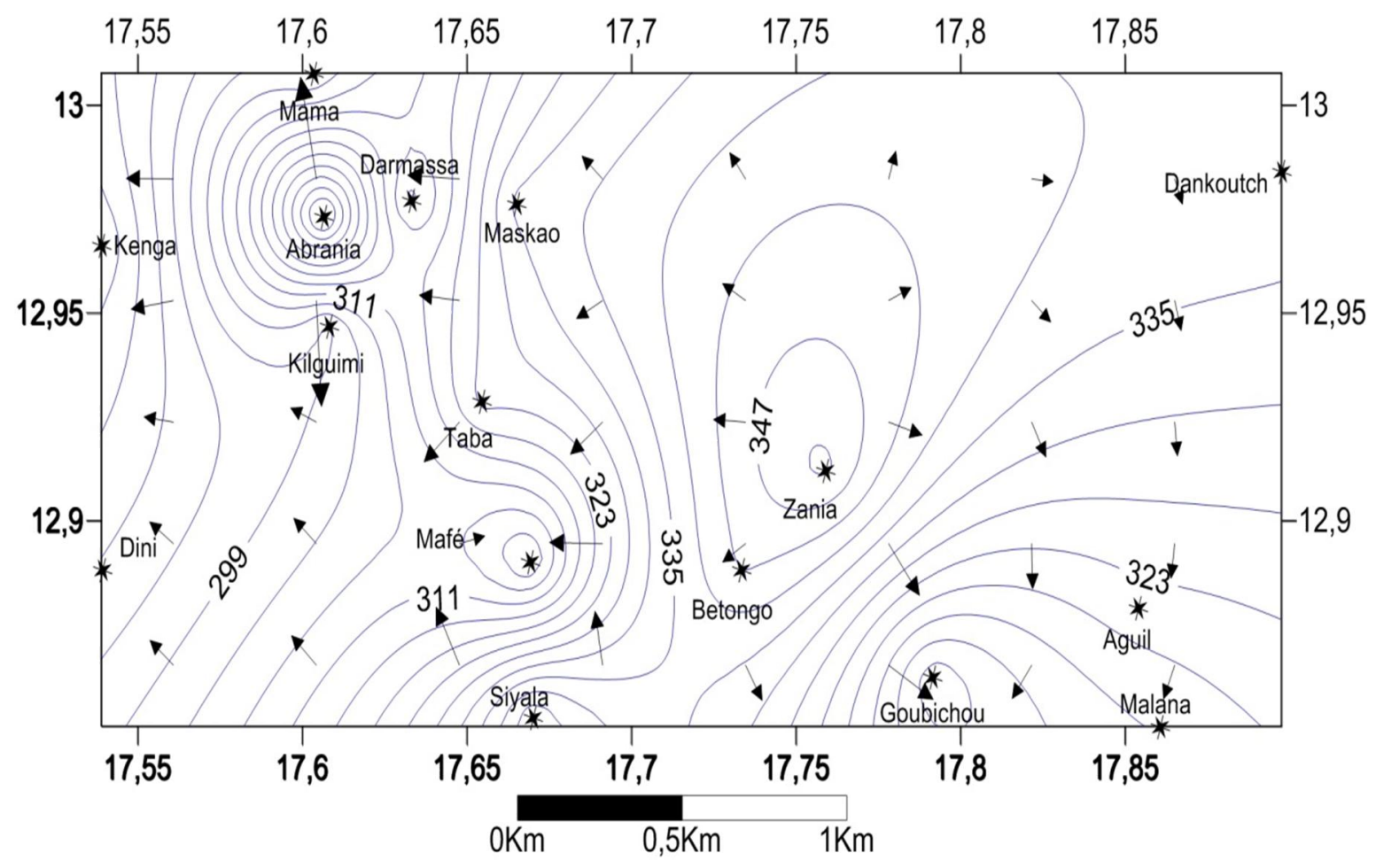

Figure 3 : Carte piézométrique du secteur d'étude.

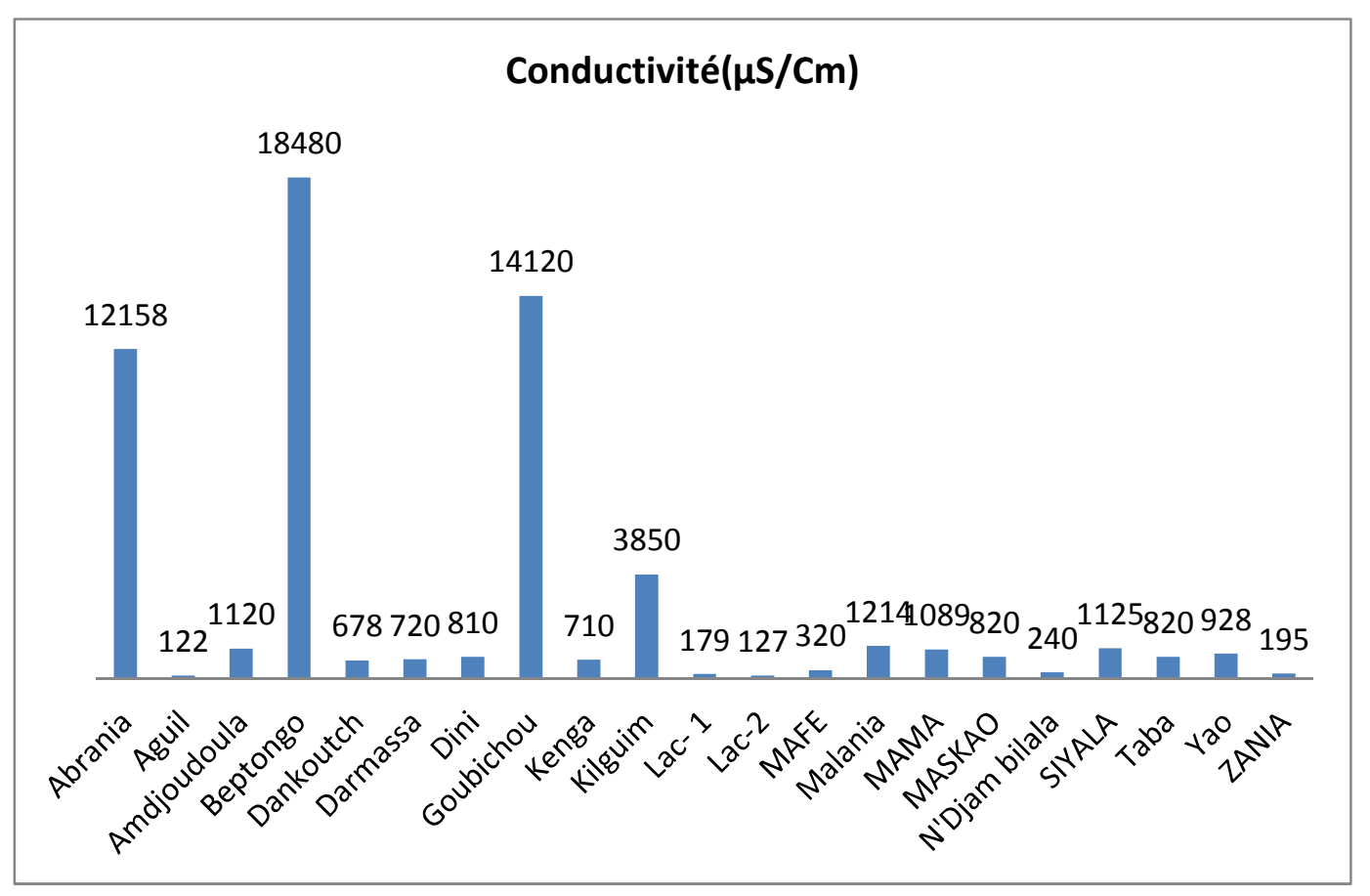

Figure 4: Histogramme des conductivités électriques. 


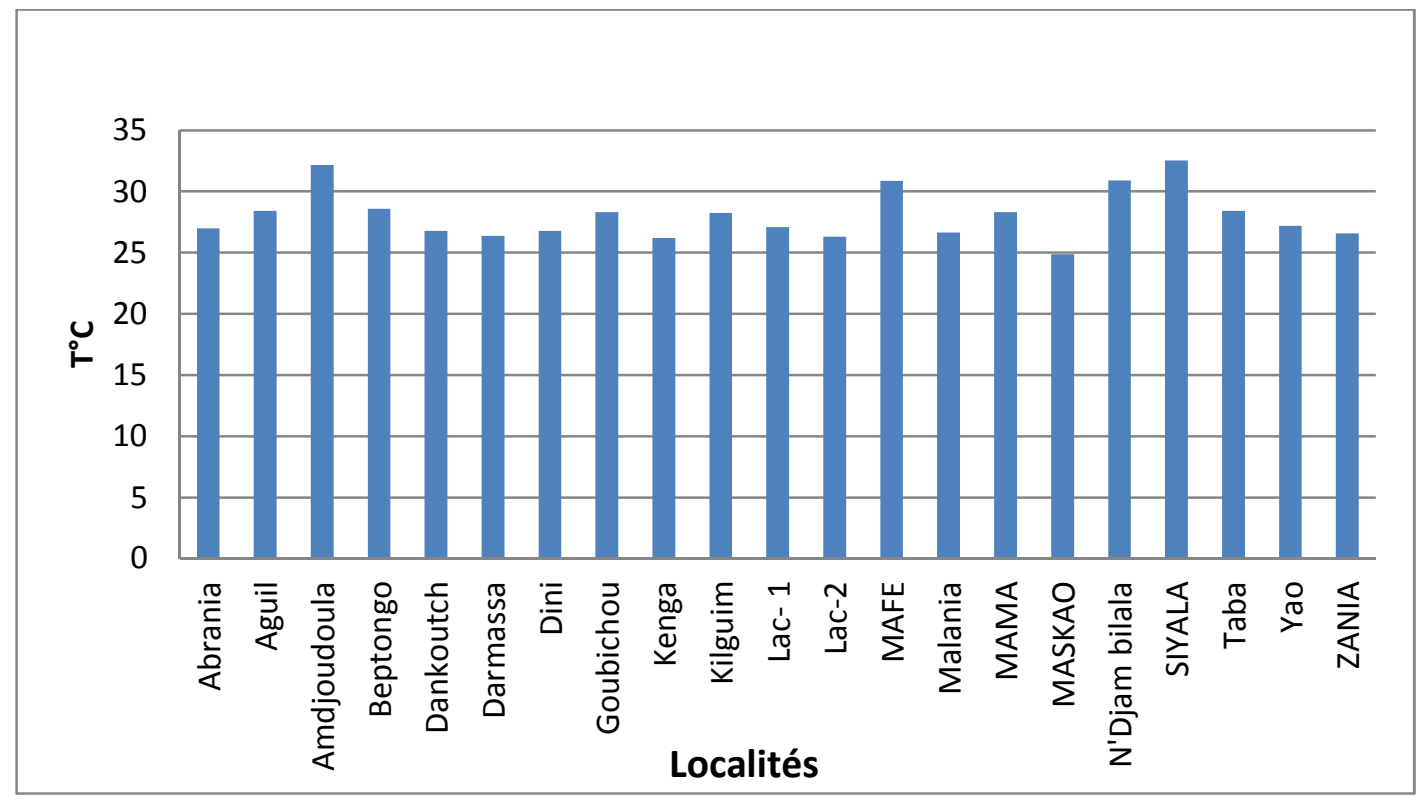

Figure 5: Histogramme des températures.

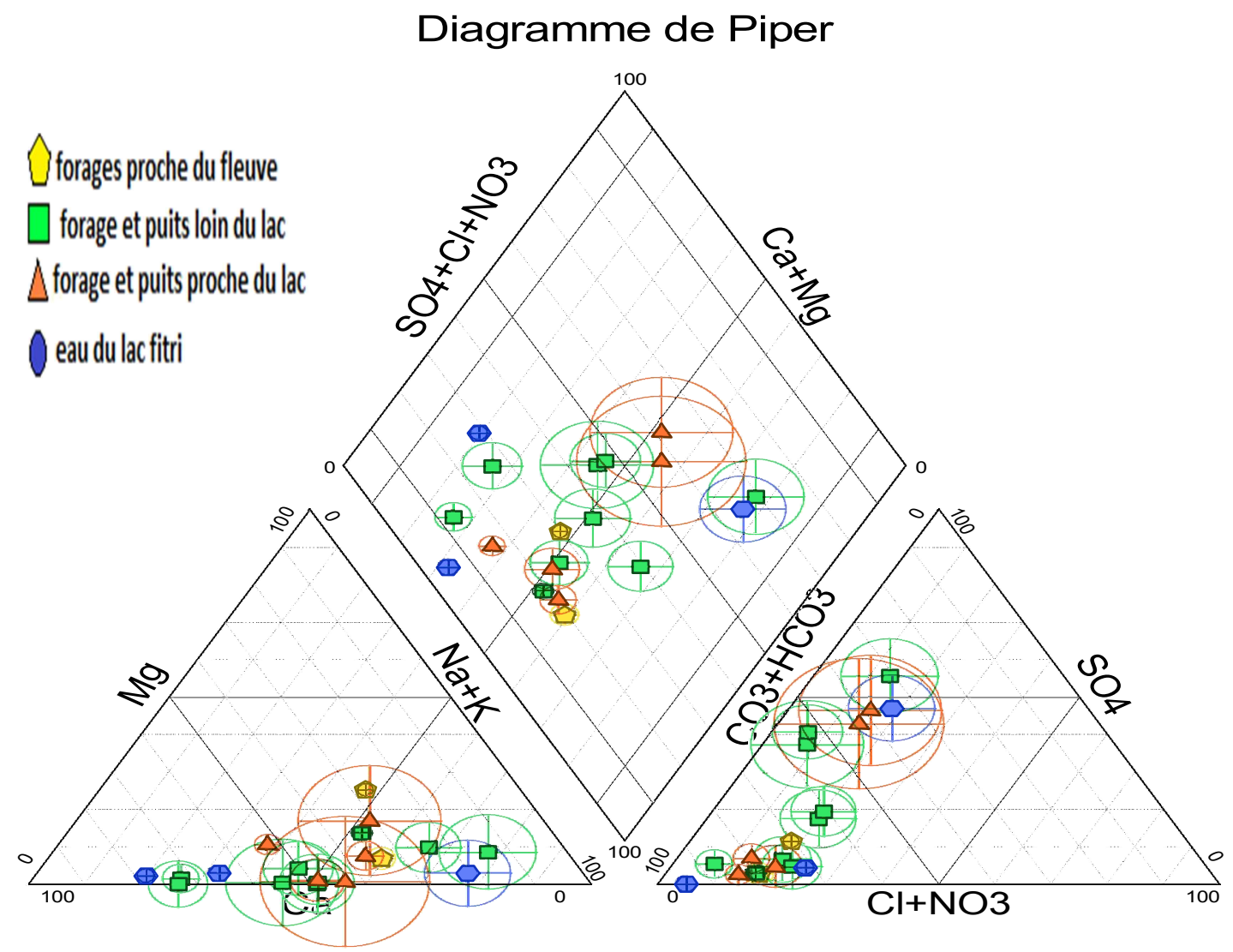

Figure 6: Diagramme de piper des eaux souterraines et de surface (région de Yao). 


\section{DISCUSSION}

La cartographie des aquifères et la mesure de niveaux piézométries permettent de déterminer les axes d'écoulements souterrains possibles (Castany, 1982). La carte piézométrique présente des courbes isopièzes. L'orientation des lignes de courant donne une vue globale sur le sens de l'écoulement des eaux souterraines et l'inventaire des ressources potentielles. En effet, cette carte piézométrique a mis en évidence les relations qui existent entre les eaux souterraines et le lac Fitri. Ainsi donc, l'analyse des données physico-chimiques et chimiques, notamment la répartition des aquifères et la cartographie des surfaces piézométriques a permis de rechercher des relations entre les écoulements souterrains et le lac.

Cette carte montre quatre zones de dépressions. A Syala, Goubichou et Mafé et deux .zones de partage ou dôme à Abrania et Zania qui contribuent à l'alimentation de la nappe.

Etant donné la capacité d'une eau à conduire le courant électrique, la conductivité électrique est directement liée à la force ionique qui découle des formations traversées par cette eau dans le bassin hydrogéologique ainsi que le temps de séjour dans le réservoir. Elle donne ainsi une bonne appréciation sur la minéralisation globale de cette eau. En effet, les valeurs de la conductivité électrique relevées dans la zone d'étude sont très hétérogènes et comprises entre $122 \mu \mathrm{S} / \mathrm{cm}$ et $18480 \mu \mathrm{S} / \mathrm{cm}$. Les plus faibles sont mesurées à Aguil, Mafé et Lac Fitri et les plus élevées sont mesurées à Beptongon, Abrania et Goubichou. Cette conductivité augmentant dans le sens de l'écoulement a permis de conclure qu'il s'agit de l'influence de la nature lithologique plus ou moins évaporitiques. Cette augmentation serait due, d'une part, au phénomène d'évaporation lié aux faibles gradients hydrauliques et d'autre part, à la dissolution des arènes granitiques et des couches évaporitiques. Ce résultat est en accord avec les travaux de Ketchemen-Tandia 2011 qui stipule que les conductivités très élevées dépassant $1000 \mu \mathrm{S} / \mathrm{cm}$ sont dues à priori à des facteurs localisés, telle la présence de décharge, la proximité des latrines et la faible épaisseur de la zone non saturée. Les gammes de variation de conductivité du lacFitri plus large sont assez hétérogène (Scuster et al., 2015).

Les pH mesurés des différents points d'eaux sont peu hétérogènes et se situent autour de la neutralité. Ils varient entre 6,34 à 8.13 avec une moyenne de 7.02. Les valeurs les plus faibles sont observées dans la zone de Beptongo et les plus élevées dans les zones d'Abrania. Les pH les plus élevés sont caractéristiques dans les eaux bicarbonatées sodiques et se situent au centre de la dépression. Ce résultat est similaire à celui de Fréderic (2000) qui a montré que cette augmentation serait due à une progression du faciès des eaux qui passe de bicarbonaté calcique au bicarbonaté sodique.

Dans la zone d'étude, les eaux souterraines présentent une température moyenne de $27,90^{\circ} \mathrm{C}$. Elles varient de 24,87 ${ }^{\circ} \mathrm{C}$ les plus basses à Maskao, à $32,17{ }^{\circ} \mathrm{C}$ les plus élevées à Amdjoudoula. Ces valeurs semblent être régies par les réactions physicochimiques exothermiques telles que l'oxydation et la réduction.

Pour la chimie des eaux, au total, 21 analyses chimiques ont été traitées pour constituer la distribution de faciès chimiques au sein de l'aquifère du quaternaire. Trois (3) faciès chimiques ont été mis en évidence dans le diagramme de Piper. On rencontre dans la quasi-totalité des eaux de la nappe, les faciès bicarbonatés calciques. Ceci peut être influencé par la prédominance des carbonates issus des eaux de surface. Par ailleurs, la dominance remarquable des teneurs en calcium sur celles en magnésium pourrait provenir d'une solubilisation préférentielle des minéraux de calcites riches en calcium par rapport aux dolomites. Les faibles concentrations $\left(\mathrm{Mg}^{2+} \mathrm{Ca}^{2+}\right)$ par rapport aux concentrations en $\mathrm{HCO}^{3-}$ permettent d'envisager un échange de base entre les ions calcium issus de la dissolution du gypse et les ions sodium des différents matériaux argileux de la masse sédimentaire. 
Les Faciès bicarbonatés sodiques sont rencontrés à l'Est de la zone d'étude. La présence de ce type de faciès bicarbonatés sodiques pourrait s'expliquer par la dissolution des formations hydrogénocarbonates de sodium ou des roches évaporitiques tel que le natron. Les Faciès sulfaté-sodiques sont rencontrés beaucoup plus au Nord de la zone d'étude. Pour ce faciès, les concentrations en sulfates et en sodium sont assez variables. Elles varient de 0,1 à 75,98 meq/l avec une moyenne de 15,66 meq/1 pour le sulfate, de 0,27 à 141,35 méq/1 avec une moyenne de $22,31 \mathrm{meq} / 1$ pour le sodium. La présence de faciès semble avoir une relation linéaire, argumentant la possible dissolution de gypse. Les coupes lithologiques sont très rares sur la région mais les faciès évaporitiques y sont fréquemment représentés (Hamid, 2010 ; Attaib, 2013). Ce qui est en accord avec l'hypothèse de Schneider (1992) effectués sur le bassin du Lac-Tchad. En revanche, l'étude de Schneider (2001) sur la géologie et l'hydrogéologie du Tchad met en évidence des séries de gypse du Moji. Cette série est repérée à l'Est du Lac du Tchad. Les nitrates, de manière générale, font partie des éléments permettant d'identifier une pollution diffuse d'origine agricole (Bouziane et Labadi, 2009). En effet, les valeurs en nitrate indiquées dans le Tableau 2 varient de 1,3 à $12 \mathrm{mg} / \mathrm{l}$. Ces valeurs sont dans la norme de l'OMS qui est de l'ordre de $50 \mathrm{mg} / \mathrm{l}$. Par contre, le chlore prend son origine dans des diverses eaux. Les teneurs en chlorures dans les eaux souterraines du secteur d'étude varient dans une large gamme entre $0,31 \mathrm{meq} / 1$ à 46,54 méq/l. Les excès en calcium et en sodium dans le diagramme de piper pourrait s'expliquer par des échanges de bases complexes entre ces deux ions au sein des matériaux argileux.

Dans l'ensemble, la qualité des eaux des nappes est bonne, mais plus minéralisée que celle du lac et nécessite, dans certains cas, un traitement spécifique avant approvisionnement (Ghebouli et al., 2008). Cette minéralisation des eaux souterraines amène les habitants de Yao à préférer l'eau du lac, moins salée que à celle des puits à pompe installés dans les villages, en dépit de sa potabilité douteuse (Schuster, 2002; Abdallah, 2013), pour éviter les maladies, sous-entendues urinaires.

\section{Conclusion}

Cette étude répond à des préoccupations malgré quelques insuffisances, l'origine de la minéralisation et le mécanisme de recharge de la nappe ainsi que les relations eau de surface - eaux souterraines ont été mis en évidence. L'étude hydrochimique montre que les eaux bicarbonatées calciques proviennent exclusivement des zones de recharge (apports météoriques ou fluviatiles). Ces eaux subissent une évolution très tendue vers un faciès bicarbonaté sodique. La conductivité des eaux souterraines de cette zone augmente dans le sens de l'écoulement. Ce qui a permis de dire qu'il s'agit de l'influence de la nature lithologique, à des facteurs localisés, telle la présence de décharge, la proximité des latrines et la faible épaisseur de la zone non saturée. L'étude piézométrique, malgré toutes ses incertitudes, délivre quelques indices quant à la dynamique de la nappe phréatique. La carte piézométrique a montré quatre zones de dépressions. A Syala, Goubichou et Mafé et deux zones de partage ou dôme à Abrania et Zania qui contribuent à l'alimentation de la nappe. La recharge de la nappe par le lac, les fleuves et les rivières est donc très liée aux conditions climatiques.

Les minéraux sont des éléments importants de l'eau potable et ont un impact sur la santé à la fois direct et indirect. L'évidence suffisante est maintenant disponible pour confirmer qu'une certaine quantité minimale de minéraux dans l'eau est désirable, puisque leur déficience a beaucoup d'effets négatifs sur la santé: maladies et agressions possibles d'éléments toxiques et de bactéries.

Le processus de minéralisation des eaux sur l'ensemble de la zone est connu. Il reste néanmoins à indiquer l'origine de la salinité des eaux. 


\section{REMERCIEMENTS}

Nous remercions l'Université Ouaga 1 Pr. Joseph Ki-ZERBO; le Laboratoire Géoressources de l'Université Ouaga 1 Pr. Joseph Ki-ZERBO; le Laboratoire National de l'Eau pour les efforts consentis dans la réalisation de cette étude.

\section{REFERENCES}

Abdallah MN. 2013. Caractérisation et fonctionnement du système aquifère de Haraz Al - Biar (Nord Chari Baguirmi), Master, Université de N'Gaoundéré; p.78-83.

Abderrahmane H. 2012. Etude du fonctionnement hydrogéochimique $\mathrm{du}$ système aquifère du Chari Baguirmi (République du Tchad). Thèse doctorat, Université de Poitiers, p114-119; 125 129.

Allison GB, Barnes CJ, Hughes MW. 1983. The distribution of deutérium and 180 in dry soils. J. Hydrology, 64: 377-397.

Aïssata BH. 2010. Aquifères superficiels et profonds et pollution urbaine en Afrique, Thèse doctorat Université Abdou Moumouni de Niamey (Niger), p.111151.

Aranyossi JF, Ndiaye B. 1993. Etude et modélisation de la formation des dépressions piézométriques en Afrique Sahélienne. Revue des Sciences de l'Eau, p.81-96.

Attaïb AB. 2014. Caractérisation hydrochimique de la nappe quaternaire du département de Fitri (Tchad), Master, Université de N'Gaoundéré, p.30-36.

Baohoutou L, Abdoulaye B, Clobite B, Mbanghoguinan A, Issak A. 2014. Impact de la variabilité pluviométrique et de la sécheresse au sud du Tchad : effets $\mathrm{du}$ changement climatique. Revue Ivoirienne des Sciences et Technologies, 23: $13-30$.

Bessoles B, Trompette R.1980. Géologie de l'Afrique. «La chaîne panafricaine », zone mobile centrale (partie Sud) et zone mobile soudanienne, 372.BRGM, Paris.
Bichara D, Safi A, Schneider JL. 1989. La précarité ou même l'absence d'alimentation de la nappe phréatique en zone nord sahélienne. Résultat d'un quart de siècle de surveillance piézométrique. C.R. Acad. Sci. : Paris ; 309.

Bouziane MT, Labadi A. 2009. Les Eaux Profondes de la Région de Biskra (Algérie). European J. Scientific Research, 25(4): 526-537.

Castany G. 1991. Principes et Méthodes Hydrogéologiques. Dunod : Paris ; 235238.

Chemseddine F. 2009. Caractérisation Hydrogéochimique des Eaux Souterraines du Complexe Aquifère Morsott-Laouinet (Région Nord de Tébessa, Sud-Est Algérien). J. Afrique Science, 5(2) : 217 - 231.

Djoret D. 2000. étude de la recharge de la nappe du Chari Baguirmi par les méthodes chimiques et isotopiques. Thèse doctorat, Université d'Avignon, p.161-192.

Eberschweiler C. $1996 . \quad$ Contextes géologiques et hydrogéologique. In Livestock Atlas of the Lake Chad Basin. Ed. CIRAD: Wageningen; 29-32.

Fréderic H. 2000. Fonctionnement Hydrogéologique et archive palioclimatiques d'un aquifère profond méditerranéen. Thèse doctorat Université d'Avignon, p.208.

Ghebouli MS, Ben cheikh El Hocine M. 2008. Origine de la salinité des eaux souterraines- cas des hautes plaines setifiennes (Nord-Est algérien). Sciences \& Technologie, 28: 37-46.

Hsissou Y. 1996. Caractérisation des eaux de l'aquifère turonien du bassin de Tadla (Maroc) par le rapport des concentrations molaires $\mathrm{Sr}^{2+} / \mathrm{Ca}^{2+}$. Journal of Hydrology 183 (1996) 443-456.

IAE. 2009. Reference fox VSMOW2 and SLAP2 International Measurement Standards. International Atomic Energy Agency Edition: Vienna; 5p. 
http://curem.iaea.org/catalogue/SI/pdf/

VSMOW2_SLAP2.pdf

INSEED. 2009. Deuxième recensement général de la population et de l'habitat (RGPH2, 2009). République du Tchad, $88 \mathrm{p}$.

Karaki. 2006. Cahier d'histoire $\mathrm{n}^{\circ} 03$ a la découverte du Tchad; p.42.

Ketchemen-Tandia B. 2011. Déterminants Hydrogéologiques De La Complexité Du Système Aquifère $\mathrm{Du}$ Bassin Sédimentaire De Douala (Cameroun). Thèse doctorat, Université de Dakar, p.200-212.

Kusnir I, BICHARA D. 1989. Géologie, ressources minérales et ressources en 307. Eau du Tchad. 1e édition, p.33.

Moussa A. 2010. Les séries sédimentaires fluviatiles, lacustres et éoliennes des bassins du Tchad depuis le Miocène terminal. Thèse Doctorat Univ. Strasbourg, p.210-294.

Moussa B. 1998. Fonctionnement du système aquifère exploite pour l'approvisionnement en eau de la ville de Cotonou sur le littoral béninois. Impact du développement urbain sur la qualité des ressources, Thèse doctorat, Université Chec Anta Diop de Dakar (Sénégal), p.108-165.

Moupeng B. 2006. Le lac Fitri : Dynamique $\mathrm{du}$ système hydrographique quaternaire et actuel (Sahel tchadien). Thèse, Université de Provence, Avignon, 171p.

Projet SIDRAT. 2010. Pré-bilan diagnostic de la région du Batha, rapport, pp30-32.

Richard L. 2000. Sur l'origine des ions chlorures dans les eaux salines des massifs granitiques. C. R. Acad. Sci. : Paris ; 780-787

Sabljak S. 1998. Hydrochimie et Hydrodynamique de la nappe phréatique au Niger sud oriental. Mémoire master, Université Paris Sud;

Schneider JL, Wolf JP. 1992. Carte géologique et carte hydrogéologique au 1/1500000e de la république du Tchad. Mémoire explicatif. BRGM, 1(209): 415.

Schneider JL. 2001. Géologique, archéologie, hydrogéologie. $B R G M, 1: 462$.

Schneider JL, Wolf JP. 1992. Carte géologique et carte hydrogéologique au 1/1500000e de la république du Tchad. Mémoire explicatif. BRGM, 2(209): 531.

Schuster M. 2002. Sédimentologie et paléoécologie des séries à vertébrés du paléo lac Tchad depuis le Miocène supérieur. Thèse de doctorat de 1'Université Louis Pasteur, Strasbourg I, $152 \mathrm{p}$

Simler R. 2004. Diagrammes. Laboratoire d'Hydrogéologie d'Avignon, Université d'Avignon, Logiciel d'hydrochimie multilangage, distribution libre. 